\title{
Radar observations of artificial E-region field-aligned irregularities
}

\author{
E. Nossa ${ }^{1}$, D. L. Hysell ${ }^{1}$, C. T. Fallen ${ }^{2}$, and B. J. Watkins ${ }^{2}$ \\ ${ }^{1}$ Earth and Atmospheric Science, Cornell University, Ithaca, NY, USA \\ ${ }^{2}$ Geophysical Institute, University of Alaska, Fairbanks, AK, USA
}

Received: 2 March 2009 - Revised: 15 June 2009 - Accepted: 23 June 2009 - Published: 7 July 2009

\begin{abstract}
Artificial E region field aligned plasma density irregularities (FAIs) were generated using HAARP in four different experimental modes and observed with a coherent scatter radar imager located $450 \mathrm{~km}$ to the southwest where it could detect field-aligned backscatter. The experiments were conducted in July of 2008, during the Polar Aeronomy and Radio Science Summer School (PARS), during quiet conditions in the daytime when the E layer was dense and absorption was modest. The echoes observed during zenith and magnetic zenith heating experiments were deflected from their nominally anticipated horizontal positions toward the midpoint position. The occurrence of hysteresis when heating with amplitude modulated pulses implied the development of the resonance instability, although the threshold for the onset of instability appeared to be higher than what has been predicted theoretically. Heating experiments involving pump frequencies slightly above and below the second electron gyroharmonic frequency produced no significant differences in the observed echoes. Finally, heating with a pump frequency slightly above the E region critical frequency appears to have produced FAIs at two distinct altitudes where the upper-hybrid resonance condition could be satisfied.
\end{abstract}

Keywords. Radio science (Waves in plasma) - Space plasma physics (Active perturbation experiments; Waves and instabilities)

\section{Introduction}

Studies of artificial ionospheric effects in the $\mathrm{E}$ region were proposed by Bailey (1959), but Fialer (1974) was the first to actually report on $\mathrm{E}$ region artificial field-aligned ionospheric irregularities (FAIs) observed by coherent scatter radar. In the eighties, Coster et al. (1985) at Arecibo and Hibberd

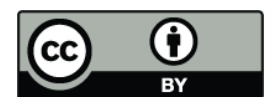

Correspondence to: D. L. Hysell (dlh37@cornell.edu) et al. (1983), Djuth et al. (1985), Hoeg (1986), and Noble et al. (1987) at Troms $\varnothing$ made further radar observations of E region FAIs. Very low heater frequencies are generally required to generate $\mathrm{E}$ layer ionospheric modifications, particularly in the absence of sporadic layers, but this capability was absent throughout the 1990s. Observations have recently become possible again due to the upgrade of HAARP, which can operate at $2.75 \mathrm{MHz}$. This is significant, since $\mathrm{E}$ region ionospheric modifications offer a means of conducting more repeatable, controlled experiments at high latitudes by comparison to their $\mathrm{F}$ region relatives by virtue of the simplicity of the radar diagnostic, which requires relatively little ionospheric refraction.

One of the methods for analyzing the volume in which FAIs are created involves imaging optical emissions. New developments in the area of aperture synthesis radar imaging allow the creation of similarly revealing images from coherent scatter radar data (Hysell and Chau, 2006). A new imaging radar system located near Homer, Alaska, offers a powerful new diagnostic for $\mathrm{E}$ region FAI research at HAARP (Hysell, 2008).

Possessing access to a low-frequency heater and appropriate diagnostics, we can now address a number of theoretical and experimental issues raised by previous experiments. Djuth et al. (1985) presented evidence of irregularity generation near the upper-hybrid resonance height, where the resonance instability is thought to produce density striations (see Mishin et al., 2005, and Gurevich, 2007, for review). The threshold pump field amplitude needed for irregularity generation was estimated experimentally by Djuth et al. (1985). Excitation thresholds for the thermal oscillating two-stream instability, which is thought to initiate the resonance instability, were predicted by Grach et al. (1977), Das and Fejer (1979), and Dysthe et al. (1983). Grach et al. (1978) and Dysthe et al. (1982) also anticipated a hysteresis effect associated with the resonance instability and wave trapping, such that irregularities can be maintained by pump waves with amplitudes below their excitation threshold. Hysteresis was

Published by Copernicus Publications on behalf of the European Geosciences Union. 


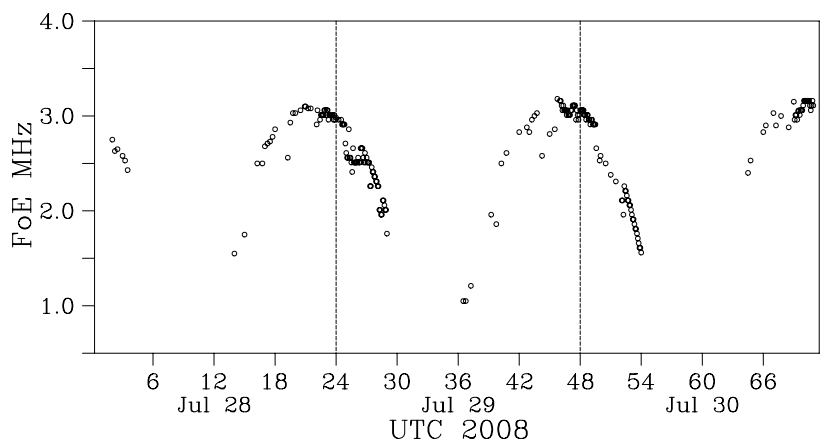

Fig. 1. FoE recorded by the HAARP ionosonde during the E region heating experiments. UT $=\mathrm{LT}+9 \mathrm{~h}$.

reported by Erukhimov et al. (1978), Jones et al. (1983), and Wright et al. (2006), although in an F region context.

Several aspects of ionospheric modifications are most intense when the heater beam is pointed toward magnetic zenith (the so-called magnetic zenith or MZ effect - see Pedersen et al., 2003, and Rietveld et al., 2003, for examples and Gurevich et al., 2002, for review). The aforementioned density striations are thought to be able to convey pump-mode radiation efficiently to altitudes above what would otherwise be a turning point, a process that can be considered as self focusing. Kosch et al. (2000) and Pedersen and Carlson (2001) have also reported on experiments involving zenith heating where the artificial emissions exhibited elongation and displacement towards the magnetic zenith. Pedersen et al. (2008) later described experiments showing displacements that were not totally consistent with the effect but similar in some respects to imaging radar results presented later in this paper.

Below, we report on recent ionospheric modification experiments involving a daytime E layer performed during midsummer with geomagnetically quiet conditions at HAARP. We assess the threshold pump wave amplitude for instability generation and look for evidence of hysteresis. We also examine differences in experiments performed with zenith and magnetic zenith heating. We also describe a serendipitous experiment that apparently led to simultaneous irregularity generation at two distinct altitudes. Experiments involving heating at frequencies just above and below the second electron gyroharmonic are also presented, although these are analyzed in detail in a companion paper (Hysell and Nossa, 2009).

\section{Observations}

The Ionospheric Research Instrument (IRI) at the High Frequency Active Auroral Research Program (HAARP) $(62.39 \mathrm{~N}, 145.15 \mathrm{~W})$ was used to generate artificial E region field-aligned density irregularities (FAIs) with its planar array of 15 by 12 low-band dipole elements. The array has a gain of $19.2 \mathrm{~dB}$ at $2.8 \mathrm{MHz}$. At this frequency, the main lobe half-power beamwidth is about 16 degrees east-west by 20 degrees north-south when pointing at zenith and can be steered up to 30 degrees away from zenith. The facility radiates 3.1 MW of peak power, giving a peak effective radiated power (ERP) of $260 \mathrm{MW}$ on zenith at this frequency.

The ionosphere over HAARP was monitored with a coherent scatter radar interferometer operating at $30 \mathrm{MHz}$ and located at the NOAA Kasitsna Bay Laboratory (KBL) $(59.47 \mathrm{~N}, 151.55 \mathrm{~W})$ near Homer, Alaska. This radar has its locus of perpendicularity in the E layer over HAARP, making it suitable for observing artificial FAIs there. The imaging radar is capable of resolving two-dimensional fine structure in backscatter from the common volume with kilometric resolution. Specifications for the radar and its operating mode were given by Hysell (2008). The HAARP ionosonde and riometer were also operating for the study.

Experiments were conducted between 28 and 29 July 2008, during the Polar Aeronomy and Radio Science (PARS) Summer School held at HAARP. Geomagnetic conditions were quiet. The experiments took place close to noontime, when the E layer critical frequency was a maximum and comparable to or greater than the lowest heating frequency used in practice at HAARP $(2.75 \mathrm{MHz})$. During the experiments, ionograms showed that the FoE (the E-region critical reflection frequency) was typically about $3.0 \mathrm{MHz}$ (see Fig. 1). The $30 \mathrm{MHz}$ riometer registered about $0.15 \mathrm{~dB}$ absorption throughout the experiments.

Figure 2 shows results from a run of the International Reference Ionosphere (IRI 2007) (Bilitza and Reinisch, 2007) with the FIRI D region model extension (Friedrich and Tokar, 2001) for the time period and geomagnetic conditions in question. The model predicts a peak $\mathrm{E}$ region density of $1.12 \times 10^{11} \mathrm{~m}^{-3}$ at about $110 \mathrm{~km}$ altitude. The model profile between $70-150 \mathrm{~km}$ can be reproduced closely by adding three Chapman functions representing the $\mathrm{D}$, $\mathrm{E}$, and $\mathrm{F}_{1}$ regions, as demonstrated by the dashed line in Fig. 2. The peak density $\left(\mathrm{m}^{-3}\right)$, peak altitude $(\mathrm{km})$, and scale height $(\mathrm{km})$ parameters used for the three functional forms here are $\left(5.5 \times 10^{8}, 85,13\right),\left(1.03 \times 10^{11}, 106,10\right)$, and $\left(1.3 \times 10^{11}, 150,27\right)$, respectively.

Figure 3 shows coherent backscatter for the heating experiments conducted on 29 July 2008. The echoes are depicted in range time Doppler intensity (RTDI) format, such that the brightness, hue, and saturation of the pixels reflect the signalto-noise (SNR) ratio, Doppler shift, and spectral width of the echoes according to the legend shown. The echoes correspond to heating intervals at HAARP, which began and ended on integral minutes. Note that, although the background conditions were relatively constant, the coherent scatter exhibited gradual variations in power and Doppler shift. This common phenomena can be seen most clearly in the echoes measured on 29 July, between 20:00 and 20:20 UT (second panel of Fig. 3). Here, there is a variation of $2 \mathrm{~dB}$ in intensity between the peaks. Gradual variations such as these, which 
we attribute to subtle changes in propagation conditions, the shape of the E layer, and the distance between the upperhybrid interaction height and the critical height, should be taken into account when assessing variations associated with changes in the heater mode.

Figure 3 depicts three distinct experiments run sequentially. The first experiment alternated the heating beam direction between magnetic zenith ( $14^{\circ}$ zenith, $202^{\circ}$ azimuth) and zenith, transmitting full power in ordinary mode $(\mathrm{CW})$ at $2.75 \mathrm{MHz}$ for 1-min intervals separated by 1 -min gaps. The FAIs observed between 20:00-20:22 UT migrated in range with heater pointing accordingly. Echo power is comparable for both pointing positions, although subtle differences in range extent are apparent. In particular, the range extent of the echoes is somewhat greater for zenith pointing than for magnetic zenith pointing.

Radar images are generated from six groups of spaced receiver antennas using aperture synthesis imaging techniques familiar in radio astronomy (i.e. Hysell and Chau, 2006). Although images can be constructed in three dimensions, the direction parallel to the geomagnetic field is uninformative, and so two-dimensional images in the plane perpendicular to B are constructed instead. Although it is possible to determine echo altitude using interferometry, the measurement is rather imprecise. We therefore assume a nominal echo altitude of $105 \mathrm{~km}$ to construct the 2-D images shown here.

Figure 4 show radar images of the artificial $\mathrm{E}$ region FAIs generated during magnetic zenith and zenith pointing, respectively, The color code for the images is the same as for the RTDI plot. Contours representing the HAARP radiation pattern, referred to a surface of constant altitude, are shown for either case to help guide the eye. In both cases, irregularities are mainly confined within the $-10 \mathrm{~dB}$ contours. In neither case are the irregularities centered precisely on the beam centers, however. In the magnetic zenith pointing direction (top panel of Fig. 4), the irregularities are concentrated toward the zenith direction, whereas for the zenith pointing direction (bottom panel), the irregularities are concentrated toward the magnetic zenith direction. Furthermore, small secondary scattering regions are evident in images for both pointing directions. These are offset from the main region in the approximate directions of the heater sidelobes. While they may in fact be telltale of ionospheric modifications in the sidelobes, the small regions are closer to the main region than one would expect in that case and lie approximately on the null of the main beam.

Another interesting feature of the images is the fact that the Doppler shifts of the echoes in the far side of the modified region are systematically different than on the near side, with the far-side echoes being red shifted compared to the near-side echoes. This phenomenon, which suggests outward expansion, was also noted by Hysell (2008) and seems to be present in either pointing position.

A second experiment, which took place between 20:2220:40 UT (see Fig. 3), incorporated amplitude modulation.

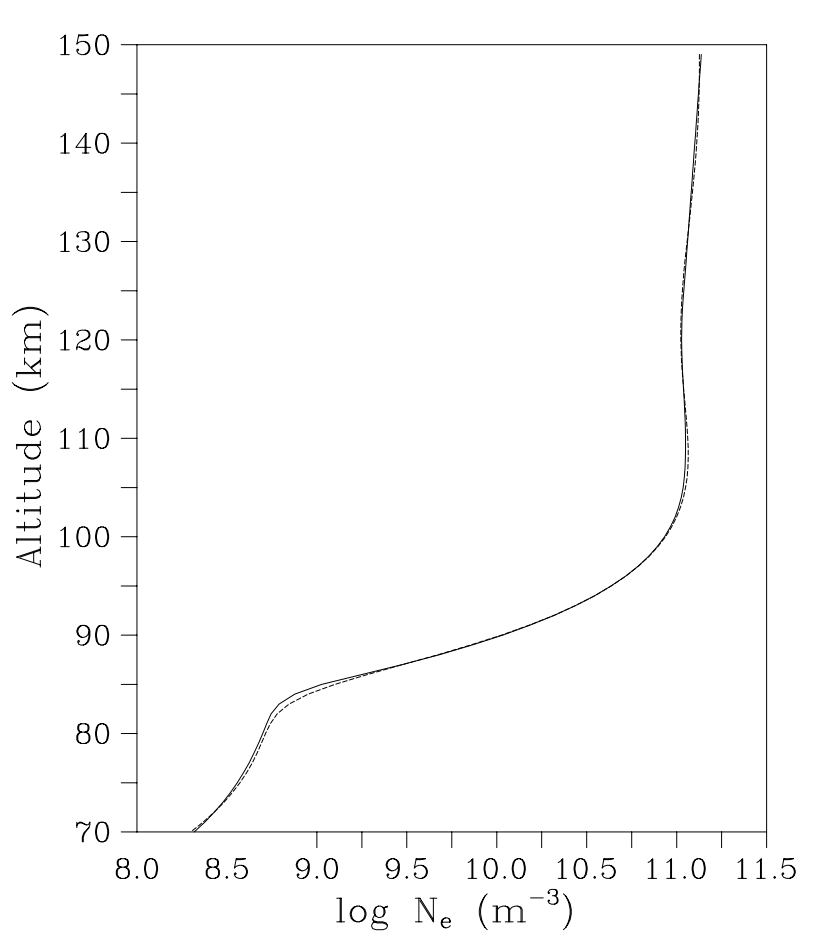

Fig. 2. Model ionosphere for the July 2009 experiments over HAARP. (solid line) IRI/FIRI model prediction. (dashed line) Reproduction of IRI/FIRI model constructed from three Chapman functions.

The heater was operated in O-mode at $2.75 \mathrm{MHz}, \mathrm{CW}$, with zenith pointing, The amplitude was modulated with a pattern that repeated every three minutes. During the first minute, the power was increased linearly from zero to full power. During the second minute, it was decreased linearly back to zero. No heating occurred in the third minute. As shown in Fig. 3, the coherent backscatter intensity waxed and waned gradually in a corresponding, if not strictly proportional, way.

A close-up view of one of the pulses (see Fig. 5) demonstrates how the FAIs are generated only after a heating power threshold is exceeded. The echo power follows the heating power thereafter, persisting almost until the heater power is off. However, there is an asymmetry in the power profile with respect to the upward and downward ramp, as manifested in somewhat different rise and fall rates. Not only the total echo power but also the range extent of the echoes appears to develop asymmetrically on the upward and downward ramps. We believe that this constitutes evidence of wave trapping and the resonance instability, a topic expanded upon below and in another manuscript (Hysell and Nossa, 2009).

A third experiment consisted of a series of 1-min. heating intervals, again separated by 1 -min. heating gaps. Omode CW heating was performed on the zenith pointing position, this time at frequencies alternating between 3.045 and $2.945 \mathrm{MHz}$. These frequencies are significant because they bracket the second electron gyroharmonic frequency, which 

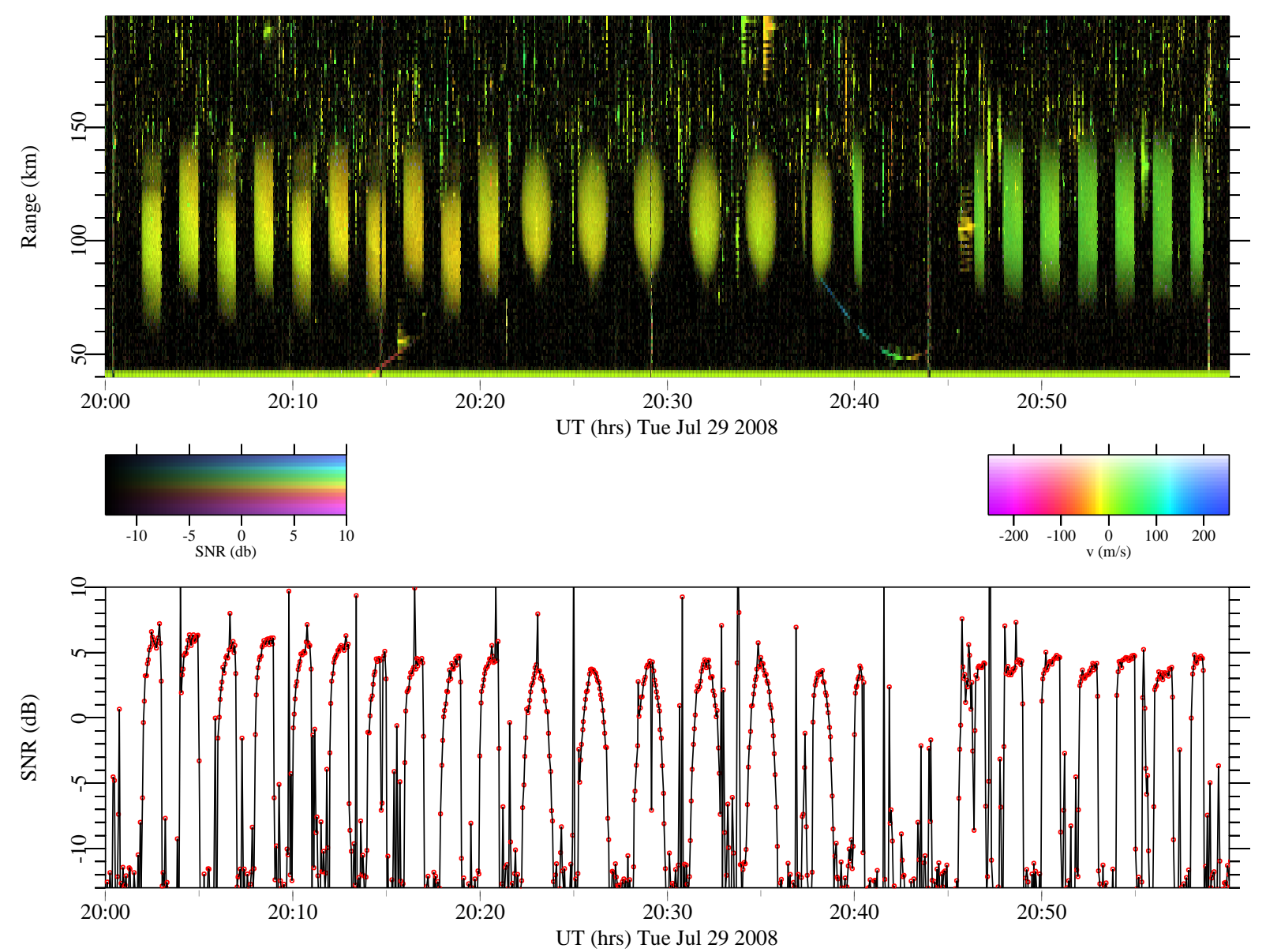

Fig. 3. Range-time Doppler intensity (RTDI) plot of backscatter from artificial E region FAIs over HAARP observed on 29 July 2008 . Here, the brightness, hue, and saturation of the pixels denote echo signal-to-noise ratio (SNR), Doppler shift, and spectral width. Note that the echoes from heater-induced FAIs are range aliased and that their true range is greater than their apparent range by $370 \mathrm{~km}$. The average signal-to-noise ratio for apparent ranges between $80-130 \mathrm{~km}$ is plotted beneath the RTDI plot. Echoes from meteor trails and aircraft are also visible in the figure. The incoherent integration time for the figure is about $3 \mathrm{~s}$. The figure depicts three distinct experiments lasting 20 min each.

is just above $3 \mathrm{MHz}$ in the $\mathrm{E}$ region over HAARP. The experiment in question was performed between 20:42-21:00, as shown in Fig. 3. The significance of this experiment is explored in detail by Hysell and Nossa (2009). Here, we note only that there appears to be no significant differences in the echoes observed for heating at the two frequencies.

A forth and final experiment was performed on 28 July using a frequency of $3.26 \mathrm{MHz}, \mathrm{CW}, \mathrm{O}$-mode, with magnetic zenith pointing. The experiment consisted of a sequence of 3 min heating intervals separated by $1.5 \mathrm{~min}$. gaps. During the gaps, HAARP emitted low duty cycle pulses which were used as a diagnostic, but these seem not to have affected the coherent scatter results. What distinguished this experiment from the others was the fact that the pump frequency was much greater than the E layer critical frequency, Apparently as a consequence, the modified region bifurcated (i.e., echoes began to be received from two regions instead of just one.)
This is clearly visible in Fig. 6, which shows what appear to be two scattering regions differing substantially in range, intensity, and Doppler shift.

The bifurcation is even more apparent in radar imagery, which is shown in Fig. 7. Here, it is clear that the echoes originated in two different regions, one near magnetic zenith $(62.2 \mathrm{~N}, 146.2 \mathrm{~W})$, and another to the northeast $(62.5 \mathrm{~N}$, $146.5 \mathrm{~W})$. We believe that the second modified region was generated on a different place on the heater ray path then the first, at a different latitude, longitude, and altitude. Note that the 105-km scattering altitude assumed in producing Fig. 7 would therefore be erroneous and that a small error in the horizontal echolocation is implied. 
Tue Jul 29 20:02:40 2008

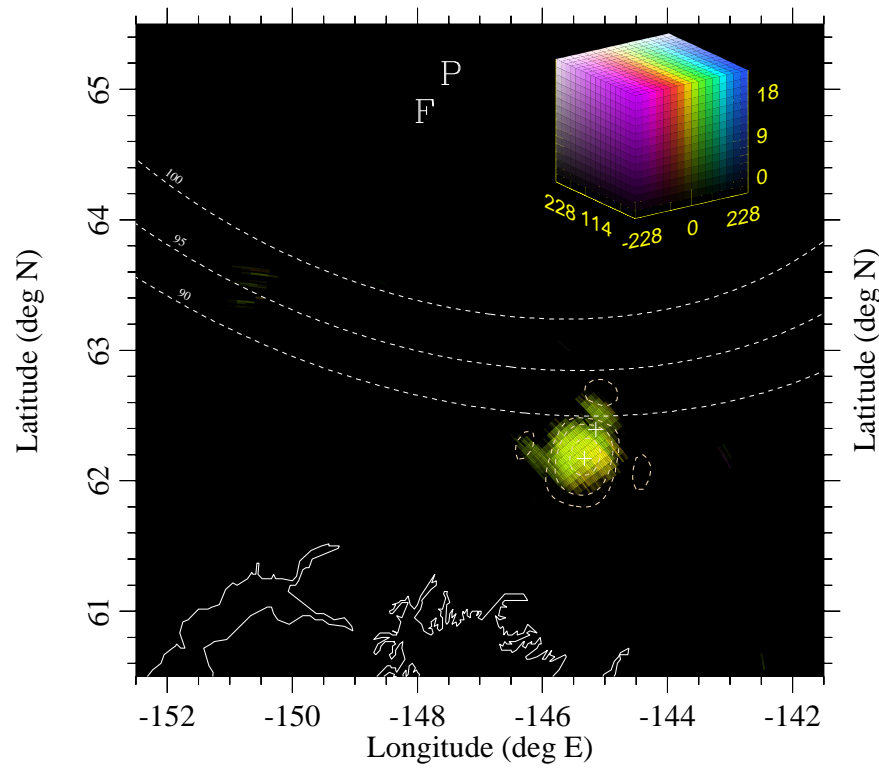

Tue Jul 29 20:04:41 2008

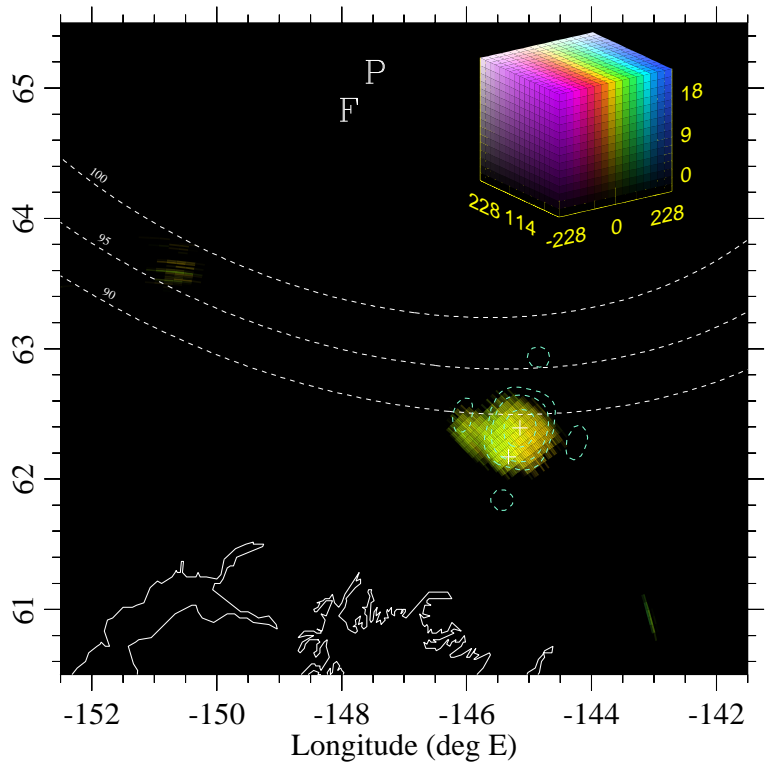

Fig. 4. Images of irregularities observed by the Homer radar. artificial E region FAIs over HAARP observed on 29 July 2008. Here, the brightness, hue, and saturation of the pixels denote echo signal-to-noise ratio (SNR), Doppler shift, and spectral width, according to the legend shown. On that legend, the vertical, horizontal, and depth axes reflect SNR (dB), Doppler shift (m/s), and spectral width (m/s), respectively. The first panel corresponds at the heater pointing to magnetic zenith and the second panel when the beam is pointing to zenith. White contours represent the loci of perpendicularity for three different altitudes, neglecting effects of refraction. Pink and cyan contours represent the $-3 \mathrm{~dB},-10 \mathrm{~dB}$, and $-15 \mathrm{~dB}$ levels of the HAARP radiation pattern for magnetic zenith and zenith pointing directions, respectively, referred to a constant altitude. The "+" symbols represent the center of the HAARP beam. The symbols P and F mark the locations of Poker Flat and Fairbanks, respectively. The image incoherent integration time is about $3 \mathrm{~s}$ for each case.

\section{Analysis}

Below, we examine and analyze the effects of changing the heater pointing position, power, and frequency on the induced artificial ionospheric irregularities and resulting coherent scatter observations.

\subsection{Zenith and magnetic zenith pointing}

The FAIs we observe are understood to be produced at the upper-hybrid resonance height (Grach et al., 1977; Das and Fejer, 1979; Grach et al., 1981; Dysthe et al., 1982, 1983). On the basis of calculations outlined below, we expect $\mathrm{O}$ mode radiation from HAARP to penetrate to this height for both zenith and magnetic zenith pointing and to generate FAIs with roughly comparable characteristics in either case. Cursory review of the data presented in the previous section supports this conclusion. In view of the importance of FAIs in the so-called magnetic zenith effect, which permits ionospheric modification at the critical reflection height even for heater pointing outside the Spitze angle, FAI generation at magnetic zenith warrants investigation nonetheless (e.g. see Isham et al., 1999.

The radar images in Fig. 4 show that the region of heaterinduced FAIs moves as the heater pointing angle changes from zenith to magnetic zenith but not in a way consistent with a simple searchlight analogy. The region is deflected from the searchlight spot toward magnetic north for magnetic zenith pointing and toward magnetic south for zenith pointing, such that the deflection is always toward the midpoint. Additionally, regions of FAIs apparently associated with the heater sidelobes follow the main FAI region, only always appearing closer to it than the HAARP radiation pattern would predict. Other deflection phenomena have been reported for airglow observations associated with the magnetic zenith effect, although it is not clear what if any relevance those observations have to the present ones (e.g. Gurevich et al., 2002; Pedersen et al., 2003, 2008). Tereschenko et al. (2006) reported that the downshifted peak emission in SEE (stimulated electromagnetic emission) experiments conducted with the EISCAT HF facility were received from angles close to magnetic zenith over a broad range of pumping angles.

A simple geometric construction due to Poeverlein and described by Budden (1988) based in the ionospheric index of refraction $n(f, \theta)$ elucidates the propagation of the pump electromagnetic wave. The index of refraction is given by the Appleton-Hartree equation:

$n^{2}=$ 


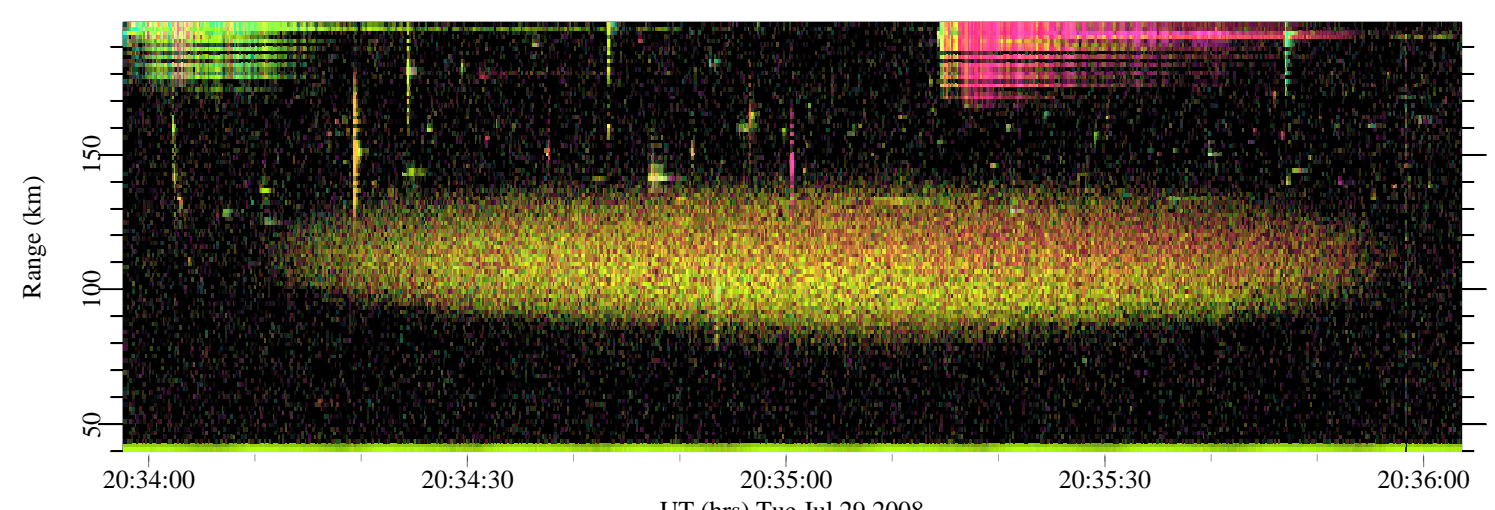

UT (hrs) Tue Jul 292008
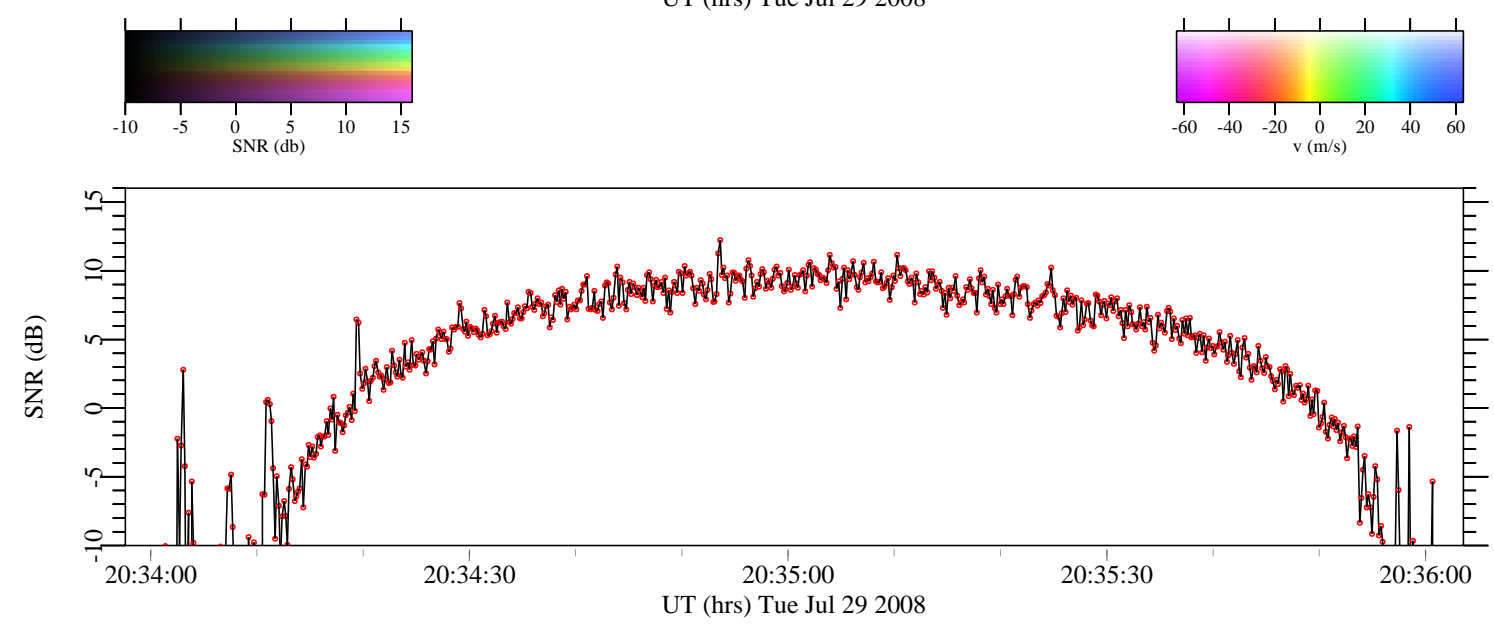

Fig. 5. RTDI plot during a triangular ramp heating pulse. Heater power increased linearly from 20:34-20:35 and then decreased linearly from 20:35-20:36. Echoes at extreme range are from long-lived meteor trails. The incoherent integration time is about $0.16 \mathrm{~s}$.

$$
1-\frac{X}{1-i Z-\frac{Y^{2} \sin ^{2} \theta}{2(1-X-i Z)} \pm \sqrt{\frac{Y^{4} \sin ^{4} \theta}{4(1-X-i Z)^{2}}+Y^{2} \cos ^{2} \theta}}
$$

where $X \equiv \omega_{p}^{2} / \omega^{2}, Y \equiv \Omega / \omega$, and $Z \equiv \nu / \omega$, and where the plus (minus) sign corresponds to $\mathrm{O}(\mathrm{X})$ mode. A vector space can be defined in terms of the coordinates $n_{x}=n \sin \theta$ and $n_{z}=n \cos \theta$, where $\hat{z}$ is the direction of the geomagnetic field, $\hat{x}$ is the transverse direction, and where $\theta$ is the angle between the pump wavevector (the wave normal direction) and the geomagnetic field. Isosurfaces of the index of refraction plotted in this space, or index of refraction surfaces, govern the direction of the pump mode ray normal, which can be shown to be everywhere perpendicular to the surfaces (e.g. Budden, 1988).

Example O-mode index of refraction surfaces for different values of $X$ and for $Y=0.5$ are show in the top panel of Fig. 8 . In this figure, the coordinate axes have been rotated $\chi=14^{\circ}$ in accordance with the magnetic zenith angle over HAARP. The $n_{z}$ and $n_{x}$ axes are the major and minor axes of the index of refraction surfaces, respectively. A wave obeying Snell's law as it propagates through a vertically stratified ionosphere can be represented by a vertical line through the figure. A wave launched from the ground toward zenith follows a ver- tical line passing through the origin. Different take-off zenith angles, $\theta_{\circ}$, correspond to lines with different abscissas. In order for an O-mode wave to propagate to the reflection height, it must have a small enough take-off zenith angle to intercept the $X=1$ line (the Spitze) in Fig. 8. Neglecting collisions in Eq. (1), this implies that $\sin \left(\theta_{\circ}\right) \leq \sqrt{\frac{Y}{1+Y}} \sin \chi$, which is the condition defining the Spitze angle. The Spitze angle is approximately $8^{\circ}$ for the experiments in question here. Most of the irregularity-filled region created by zenith heating lies within the Spitze cone.

A similar calculation can be performed to find the largest take-off zenith angle for which an O-mode wave launched from the ground will propagate so as to intercept the index of refraction surface corresponding to the upper hybrid resonance condition, $X=1-Y^{2}$. We have solved for this condition numerically and plotted the angle in question as a function of $Y$ in the bottom panel of Fig. 8. For the experiments described here, for which $Y \sim 0.5$, all of the pointing positions available at HAARP, including the magnetic zenith position, lie within the range of angles indicated by the curve.

Figure 8 also illustrates the difficulty of explaining the deflections of the irregularity-filled regions from the simple 


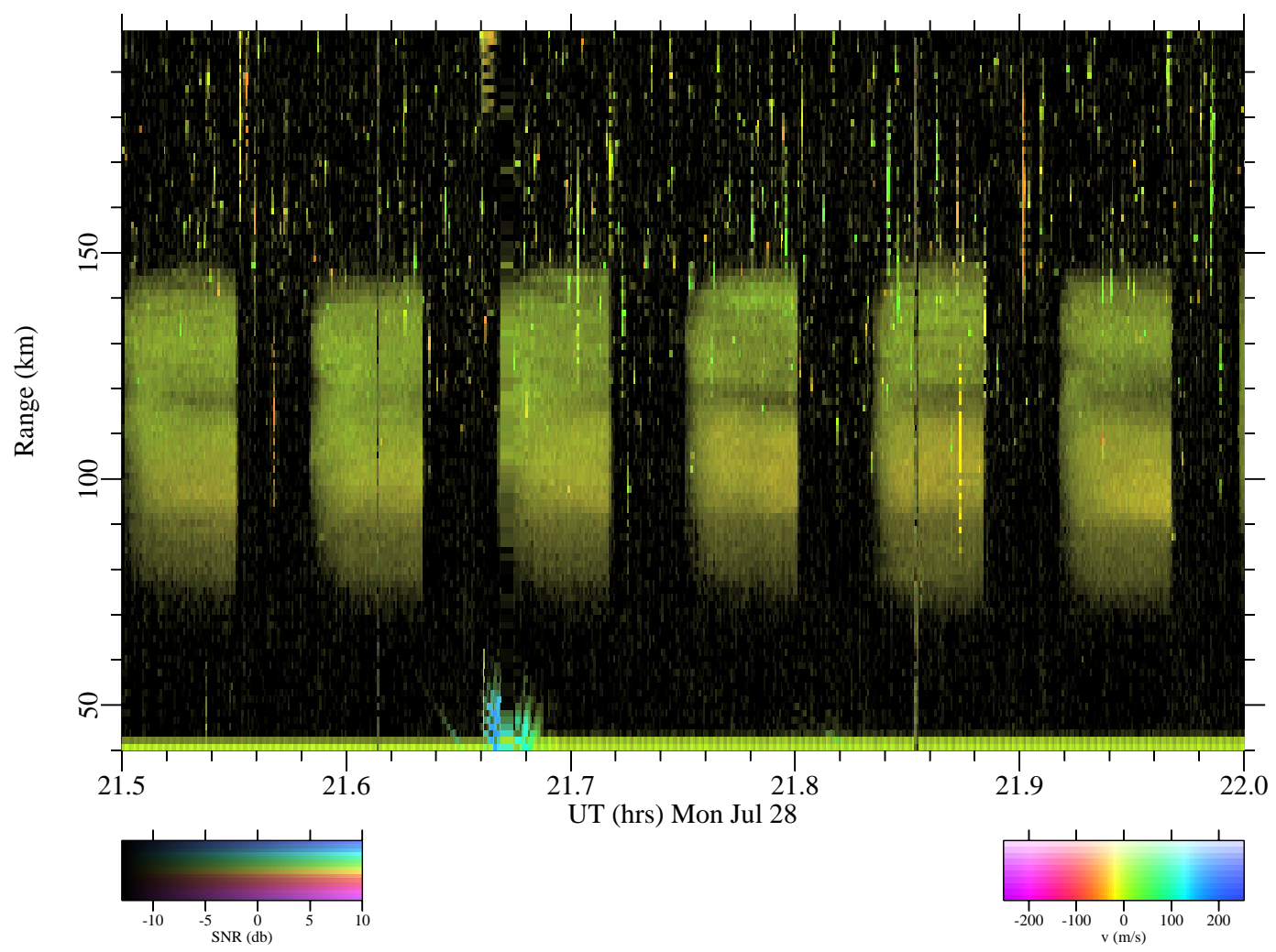

Fig. 6. RTDI plot of backscatter from artificial E region FAIs over HAARP observed on 28 July 2008.

searchlight picture. Arrows in the top panel of the figure represent the ray-normal direction of the waves along their propagation paths. Differences between the ray-normal and wave-normal directions imply deflection. The arrows show how $\mathrm{O}$-mode rays reflected at the critical height deflect so as to have ray normals perpendicular to the geomagnetic field on the Spitze. Off of the Spitze, the deflection of refracted waves is still towards perpendicularity, although the bending angles involved are much less severe. In either case, the net deflection is toward the magnetic pole. While this is consistent with our observations of artificial FAIs created with magnetic zenith heating, it is inconsistent with zenith heating results, where the deflection is toward magnetic south.

\subsection{Hysteresis and striation formation}

Figure 9 is a composite plot representing the coherent echo backscatter detected by the $30 \mathrm{MHz}$ radar as a function of the fractional heating power radiated by HAARP. Different colors are used to represent the five complete heating cycles performed. Up and down arrows indicate whether the power was increasing or decreasing. The black curve illustrates what a linear relationship would look like on the semi-logarithmic plot. While some variability is evident from cycle to cycle, the figure shows hysteresis in the heating cycles, viz., a history dependence in coherent echo strength versus heater

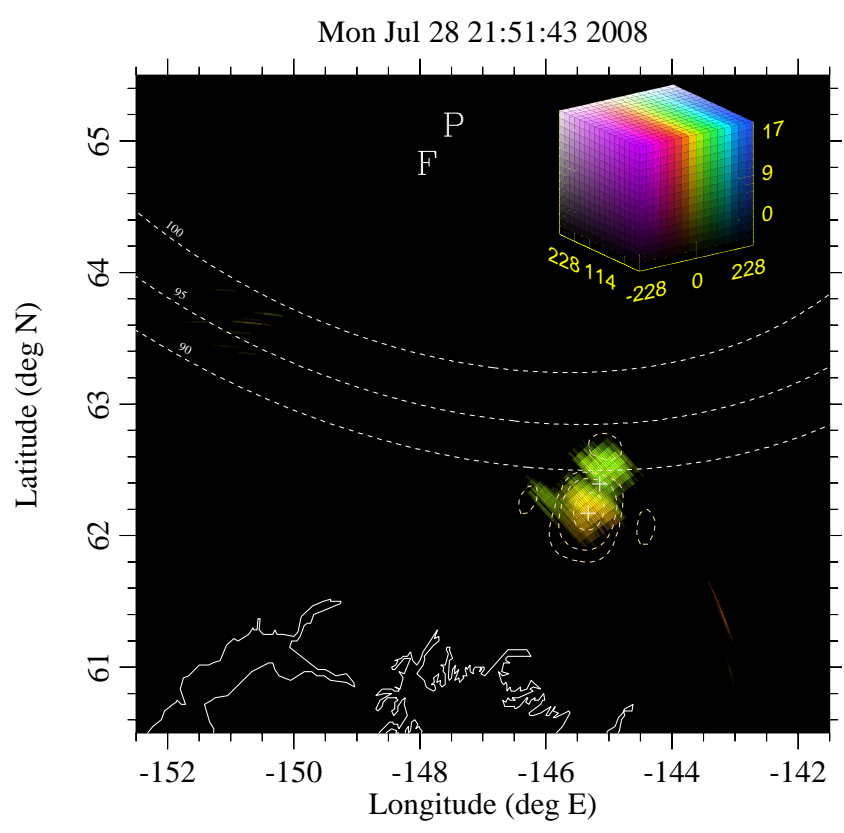

Fig. 7. Images of irregularities observed on July 28, 2008. Contours representing the HAARP radiation pattern for magnetic zenith pointing are shown. 


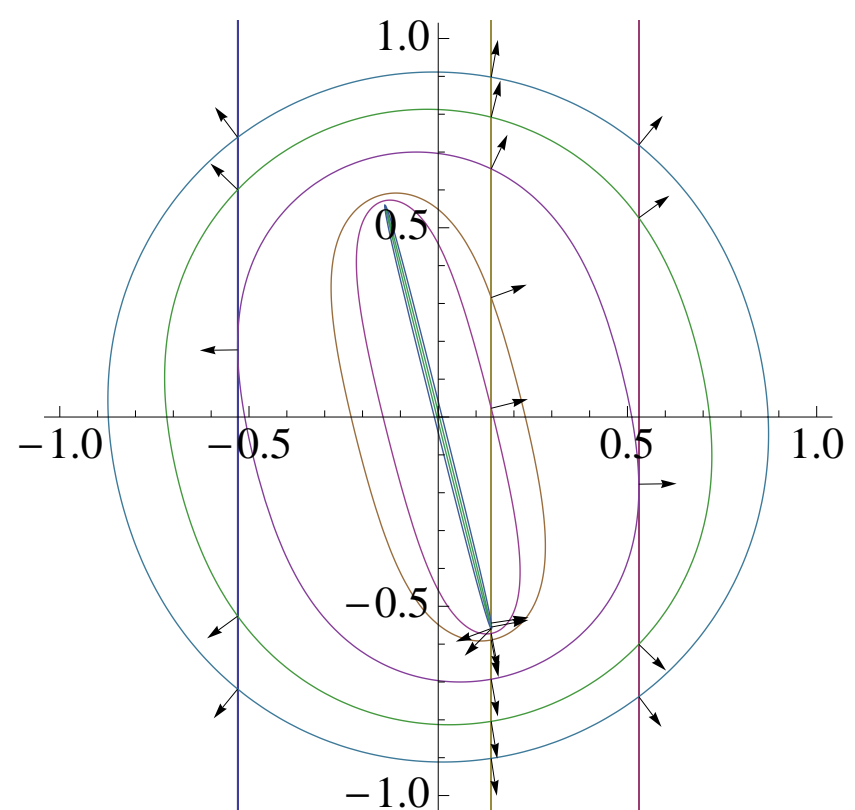

Zenith Angle (deg)

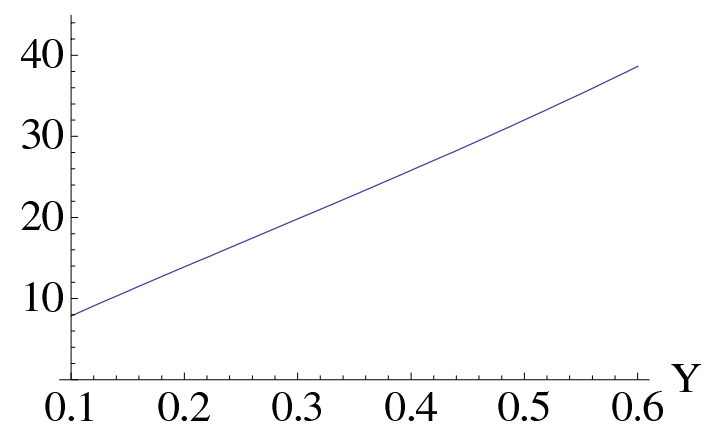

Fig. 8. O-mode index of refraction surfaces for $Y=0.5$ and $X=\{1$, $0.95,0.75,0.5,0.25\}$, drawn in the plane of the magnetic meridian. The magnetic zenith angle is $14^{\circ}$. The vertical lines represent waves following Snell's law (see text). (Bottom) Maximum zenith angle for upper-hybrid resonance as a function of $Y$.

power. In view of Fig. 6, it appears that irregularities are not produced until the heater power reaches about one fifth its maximum while irregularities can be maintained virtually until the time heating is discontinued.

Grach et al. (1978) and Dysthe et al. (1982) predicted this kind of hysteresis, arguing that it should be possible to maintain irregularities with less power than is necessary to excite them because differential heating by the the resonance instability increases in efficiency with the intensity of the irregularities. Some preliminary evidence for their hypothesis was available at that time (Erukhimov et al., 1978; Stubbe et al., 1982; Kopka et al., 1982). Additional evidence of hysteresis has since been provided by Jones et al. (1983) and Wright et al. (2006).

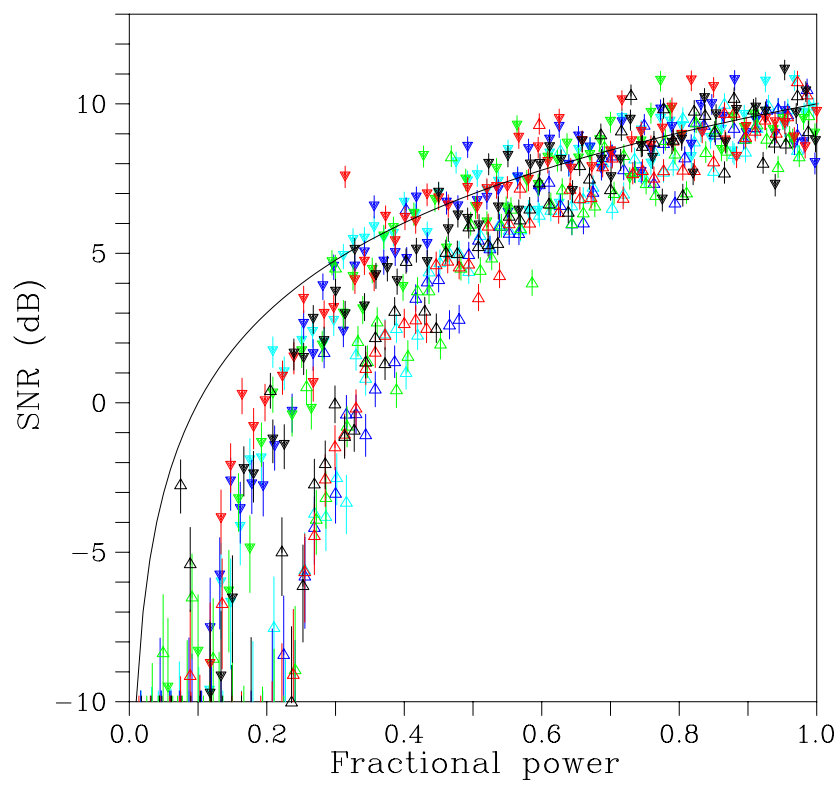

Fig. 9. Hysteresis curves (coherent scatter SNR versus fractional heater power) derived from five sequential heating events like the one in Fig. 5. The incoherent integration time for each value is $1 \mathrm{~s}$. The solid curve represents a linear relationship and is for reference.

Since the thermal oscillating two stream instability is generally believed to initiate the resonance instability, the former process should set the pump mode intensity threshold for ultimate irregularity generation. Grach et al. (1977), Das and Fejer (1979), and Dysthe et al. (1983) estimated the pump mode intensity threshold in question, including provisions for $\mathrm{E}$ region calculations. Below, we compare the prediction of Dysthe et al. (1983) with experimental results.

For our calculations, we assume an electron-neutral collision frequency of $5 \times 10^{4} \mathrm{~s}^{-1}$ at an altitude of $100 \mathrm{~km}$ with a scale height of $6.5 \mathrm{~km}$. The total absorption can be calculated using this estimate together with Eq. (1) and the electron density profile shown in Fig. 2. Such a calculation gives $\int d z 2 k \Im(n(z))=0.0335$, where the integration is over the altitudes shown in Fig. 2 and the wave frequency is set to $30 \mathrm{MHz}$. The corresponding attenuation of $0.15 \mathrm{~dB}$ agrees with the riometer observations made during our experiments, supporting the efficacy of the electron density and collision frequency profile estimates.

The HAARP effective radiated power of $260 \mathrm{MW}$ implies an electric field intensity of about $1.75 \mathrm{~V} / \mathrm{m}$ on average over the illuminated area at a reference altitude of $70 \mathrm{~km}$. Irregularities were observed when the heater power exceeded about one fifth its maximum, the corresponding electric field amplitude being about $780 \mathrm{mV} / \mathrm{m}$ at $70 \mathrm{~km}$. Estimating the field intensity at the upper-hybrid resonance height, which would have been between $98-99 \mathrm{~km}$ for heating at $2.75 \mathrm{MHz}$ according to Fig. 2, requires that magnetoionic effects and classical absorption be taken into account. A formalism for 
doing so was provided by Thidé and Lundborg (1986), who applied the uniform approximation technique to a full-wave treatment to the problem of electromagnetic wave propagation in inhomogeneous, anisotropic, lossy media. Their formulation reproduces Airy swelling and dissipative damping, providing solutions for the vector wave electric field components in a vertically stratified ionosphere in situations where one or two turning points exist. Their formulation also takes into account the $r^{-1}$ dependence in field amplitude.

Electric field solutions for conditions representative of our zenith heating experiments at $2.75 \mathrm{MHz}$ are shown in Fig. 10. The curves represent the standing wave envelopes for the three components of the electric field, as indicated in the figure caption. The curves illustrate how collisional damping largely counteracts Airy swelling and leads to attenuated field amplitudes except in the parallel component. So severe is the absorption that the reflected wave is nearly suppressed. The standing wave is, in fact, almost a purely upward propagating wave in this case, as evidenced by the fact that the wave crests and troughs have similar amplitudes. It is the perpendicular electric field component that is of interest for field-aligned irregularity generation, and we conclude from Fig. 10 that the pump mode electric field amplitude at the upper-hybrid resonance height was likely about $320 \mathrm{mV} / \mathrm{m}$ by the time the irregularities were first observed $\sim 12 \mathrm{~s}$ after heating began.

Meanwhile, the threshold electric field amplitude for thermal oscillating two stream instability at $100 \mathrm{~km}$ altitude under the conditions of our experiments predicted by Dysthe et al. (1983) is about $80 \mathrm{mV} / \mathrm{m}$. (Note that the effect of the reflected wave was neglected in our calculation in view of the preceding analysis.) In our experiments, this threshold was exceeded after about $1 \mathrm{~s}$ of heating. Djuth et al. (1985) reported a similar finding from their analysis of artificial $\mathrm{E}$ region FAI data from Scandinavia. Evaluating threshold electric field predictions made by Dysthe et al. (1983), Grach et al. (1977), and Das and Fejer (1979), they arrived at figures between about $40-90 \mathrm{mV} / \mathrm{m}$.

Comparing prediction with observation is complicated in our case by latency issues (the time it takes for irregularities to grow to reach resonance instability) and the fact that the pump power varied throughout our experiment. According to Dysthe et al. (1983), the growth time for thermal oscillating two-stream instability is inversely proportional to the fourth power of the pump electric field. Using their formalism, it is possible to estimate the number of $e$-folds occurring after threshold is reached as a function of experiment time, at least approximately. For conditions relevant for our experiments, including the rate of increase of pump power during ramp-up, irregularities could be expected to have undergone $10 e$-folds $4-5 \mathrm{~s}$ after exceeding threshold and $100 e$-folds by $10 \mathrm{~s}$. The latter figure is absurdly large, and irregularities should have been observed several seconds earlier than they were in our experiments according to theory. A possible explanation for the discrepancy is anomalous absorption, which could begin

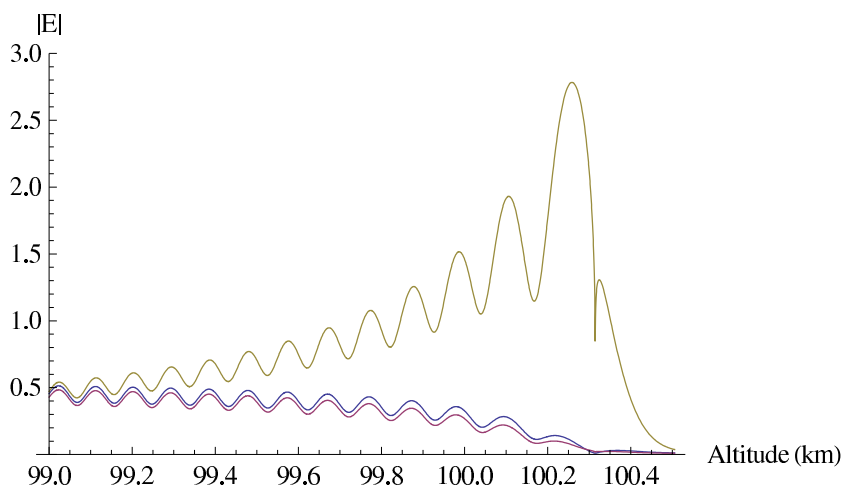

Fig. 10. Electric field amplitude for a pump wave at $2.75 \mathrm{MHz}$ propagating through the ionosphere given by Fig. 2. A magnetic declination of $14^{\circ}$ is assumed. The reflection height in this case is $100.3 \mathrm{~km}$. The perpendicular-east (magnetic), perpendicular-north (magnetic), and parallel components of the electric field are depicted in blue, red, and green, respectively. Amplitudes as a fraction of the amplitude at a $70 \mathrm{~km}$ reference altitude are represented.

limiting the pump mode electric field amplitude the moment threshold is exceeded.

Djuth et al. (1985) reported that irregularities could be sustained in their experiments by an estimated pump mode electric field amplitude of only about $100 \mathrm{mV} / \mathrm{m}$. While their experiments were run with an ERP comparable to ours, they assumed somewhat greater classical absorption and also invoked anomalous absorption in evaluating the steady-state pump electric field amplitude at the upper-hybrid resonance height. Greater classical absorption is consistent with the fact that theirs were postsunset experiments and relied on particle precipitation to provide $\mathrm{E}$ layer ionization. The effects of anomalous absorption were incorporated using the formalism of Graham and Fejer (1976) with the assumption of fully developed irregularities. That irregularities were observed in our experiments in the waning 1-2 s of heating supports the conclusion that they can be sustained by pump electric fields at the $100 \mathrm{mV} / \mathrm{m}$ level.

\subsection{Multiple echoes}

The coherent scatter data shown in Figs. 6 and 7 suggest that ionospheric irregularities were generated in two distinct regions by heating at a frequency $(3.26 \mathrm{MHz})$ above the $\mathrm{E}$ region critical frequency $(3.0 \mathrm{MHz})$. That the irregularities could have been generated at different altitudes is suggested by Fig. 2. The figure implies that the reflection height should have been around $150 \mathrm{~km}$ and that the upper hybrid resonance frequency could have been matched near $110 \mathrm{~km}$ altitude and again near about $130 \mathrm{~km}$ altitude.

The hypothesis can be tested using classical radar interferometry. Among the antenna groups used by the $30 \mathrm{MHz}$ radar imager are two which are separated by 4.5 wavelengths and aligned nearly with the line-of-sight to HAARP. The 


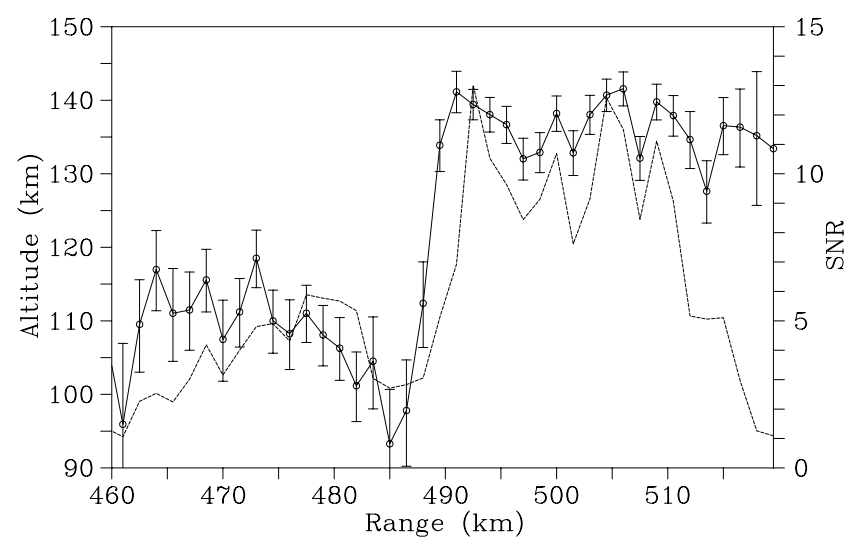

Fig. 11. Relative altitudes of two scattering regions shown in Fig. 7 estimated from interferometry using a single longitudinal baseline. The solid line with error bars represents altitude. Note that the error bars reflect statistical fluctuations only and not systemic errors. The dashed line shows the signal-to-noise ratio. Only data from the zero Doppler frequency bin were used for this calculation. The incoherent integration time is about $13 \mathrm{~s}$.

elevation angle of the backscatter and the echo region altitude can be estimated from the interferometric phase angle measured using this antenna pairing with the methodology introduced by Farley et al. (1981). The low elevation angle of the backscatter and the shortness of the interferometry baseline in this case preclude very precise altitude estimates. Uncertainty in the height of the antennas and the electrical behavior of the ground beneath them also impair the accuracy of the estimates. However, drastic variations in scatterer altitude should be apparent in interferometry phase angle data given sufficient signal-to-noise ratios and incoherent integration times.

Figure 11 shows coarse estimates of the scatterer altitude versus range for the radar echoes shown in Fig. 7. The data are derived from the zero-frequency Doppler spectral bin. Also shown is the signal-to-noise ratio in that bin. The double-humped SNR distribution clarifies the locations of the two main scattering regions. The figure shows that the more distant region is about $20 \mathrm{~km}$ higher than the less distant one. The result is roughly consistent with the electron density profile in Fig. 2 and the arguments outlined above.

A drastic difference in the altitudes of the two scattering regions also explains why their Doppler shifts differ. Whereas the ions are unmagnetized in the lower region, they are marginally magnetized in the upper region. Doppler shifts in the lower region are likely to be strongly influenced by neutral dynamics. To the extent that the electric field has influence, the component of the electric field parallel to the radar line-of-sight should be most important. In the higher region, the electric field should dominate, and the component transverse to the radar line-of-sight will be most important.
The horizontal displacement of the irregularity-filled highaltitude region magnetic northward of the magnetic zenith heating position is superficially consistent with expectations based on the index of refraction surface and ray normal theory given that the pump ray would have been deflected northward so as to be perpendicular to the geomagnetic field at the reflection height. Absorption for the $3.26 \mathrm{MHz}$ pump wave would have been less severe than at $2.75 \mathrm{MHz}$, and the significant $(\sim 25 \mathrm{~km})$ displacement suggests that irregularities might have been generated by the reflected wave. Quantitative analysis of the displacement and the pump wave ray path remains to be performed, however.

\section{Summary}

Observations of E region FAIs presented in this paper generally support prevailing theories regarding irregularity generation in ionospheric modification experiments, although a number of remaining problems and some inconsistencies are evident. Comparable irregularity features in zenith and magnetic zenith heating experiments support the predominant role of processes at the upper hybrid resonance level in irregularity generation, which is already well established. However, the horizontal distribution of the irregularities particularly in zenith heating experiments, cannot be understood readily in terms of simple ray normal theory. Detailed raytracing calculations incorporating calculations of the transverse electric field amplitude may help resolve the issue.

The observation of hysteresis in the irregularity amplitude in experiments where the heater power is ramped up and down is consistent with resonance instability theory. However, our experiments point to a discrepancy between the predicted and observed threshold and/or growth rate for thermal oscillating two-stream instability, which is thought to initiate the resonance instability. Much of the discrepancy may lie in uncertainties regarding background conditions and other physical properties at the interaction height. The E region transport coefficients in particular are not necessarily well known, and the threshold condition is very sensitive to them and a number of other parameters (e.g. E region density, temperature, scale height, collision frequency, etc.) which cannot be precisely specified. Furthermore, the role of anomalous absorption prior to the onset of the resonance instability requires further investigation. Additional experiments involving discretely-stepped heater power levels and long dwells could be used to resolve some of these issues.

A serendipitous experiments involving heating above the $\mathrm{E}$ region critical frequency appears to have produced artificial irregularities in the $E$ and $F_{1}$ regions simultaneously. Not only does such an experiment potentially provide a means of diagnosing neutral winds and background electric fields in the lower thermosphere, it also conveys information about the magnetic aspect sensitivity of the irregularities. The nominal locus of perpendicularity for the ray path from Homer to 
Gakona for a $30 \mathrm{MHz}$ radio wave is at approximately $100 \mathrm{~km}$ altitude, and the magnetic aspect angle for irregularities at $\sim 130 \mathrm{~km}$ altitude is more than one degree. Nevertheless, coherent echoes received from the higher altitude were comparable in intensity to echoes from the lower altitude. This suggests that artificially generated $\mathrm{E}$ region ionospheric irregularities have considerably broader aspect angle RMS half widths than do naturally occurring irregularities, for example. Future experiments could be used to measure the aspect angle of artificial irregularities explicitly.

Acknowledgements. The authors are grateful for help received from the NOAA Kasitsna Bay Laboratory, its Director Kris Holderied, Lab Manager Mike Geagel, and Lab Director Connie Geagel. We also appreciate helpful comments from C. La Hoz from the University of Troms $\varnothing$ who is presently on leave at Cornell. This work was supported by the High Frequency Active Auroral Research Program (HAARP) and by the Office of Naval Research and the Air Force Research Laboratory under grant N00014-07-1-1079 to Cornell.

Topical Editor M. Pinnock thanks A. Streltsov and B. Isham for their help in evaluating this paper.

\section{References}

Bailey, V. A.: Some possible effects caused by strong gyro-waves in the ionosphere, 1, J. Atmos. Terr. Phys., 14, 299-324, 1959.

Bilitza, D. and Reinisch, B.: International Reference Ionosphere 2007: Improvements and new parameters, J. Adv. Space Res., 42(4), 599-609, 2007.

Budden, K. G.: The Propagation of Radio Waves, Cambridge Univ. Press, New York, 1988.

Coster, A. J., Djuth, F. T., Jost, R. J., and Gordon, W. E.: The temporal evolution of 3-m striations in the modified ionosphere, J. Geophys. Res., 90, 2807-2818, 1985.

Das, A. C. and Fejer, J. A.: Resonance instability of small-scale field-aligned irregularities, J. Geophys. Res., 84, 6701-6704, 1979.

Djuth, F. T., Jost, R. J., Noble, S. T., Gordon, W. E., Stubbe, P., Kopka, H., Nielsen, E., Boström, R., Derblom, H., Hedberg, Á., and Thidé, B.: Observations of $E$ region irregularities generated at auroral latitudes by a high-power radio wave, J. Geophys. Res., 90, 12293-13206, 1985.

Dysthe, K., Mjølhus, E., Pécseli, H., and Rypdal, K.: Thermal cavitons, Phys. Scr. T., 2, 548-559, 1982.

Dysthe, K., Mjølhus, E., Pécseli, H., and Rypdal, K.: A thermal oscillating two-stream instability, Phys. Fluids, 26, 146-157, 1983.

Erukhimov, L. E., Metelev, S. A., Mityakov, N. A., and Frolov, B. L.: Hysteresis effect in the artificial excitation of inhomogeneities in the ionospheric plasma, Radiophys. Quantum Electron. (Engl. Trans.), 21, 1738-1740, 1978.

Farley, D. T., Ierkic, H. M., and Fejer, B. G.: Radar interferometry: A new technique for studying plasma turbulence in the ionosphere, J. Geophys. Res., 86, 1467-1472, 1981.

Fialer, P. A.: Field-aligned scattering from a heated region of the ionosphere - Observations at HF and VHF, Radio Sci., 9, 923940, 1974.
Friedrich, M. and Tokar, K.: FIRI: A semiempirical model of the lower ionosphere, J. Geophys. Res., 106(A10), 21409-21418, 2001.

Grach, S., Mityakov, N., Rapoport, V., and Trakhtengertz, V.: Thermal parametric turbulence in a plasma, Physica, D, 2, 102-106, 1981.

Grach, S. M., Karashtin, A. N., Mityzkov, N. A., Rapoport, V. O., and Trakhtengerts, V. Y.: Parametric interactions between electromagnetic radiation and ionospheric plasma, Radiophys. Quantum Electron. (Engl. Transl.), 20, 1254-1258, 1977.

Grach, S. M., Karashtin, A. N., Mityzkov, N. A., Rapoport, V. O., and Trakhtengerts, V. Y.: Theory of thermal parametric instability in an inhomogenous plasma (nonlinear theory), Sov. J. Plasma Phys. (Engl. Trans.), 4, 742-747, 1978.

Graham, K. N. and Fejer, J. A.: Anomalous radio wave absorption due to ionospheric heating effects, Radio Sci., 11(12), 10571063, 1976.

Gurevich, A. V.: Nonlinear effects in the ionosphere, Uspekhi Fizicheskikh Nauk., 177(11), 1145-1177, 2007.

Gurevich, A. V., Zybin, K. P., Carlson, H. C., and Pedersen, T.: Magnetic zenith effect in ionospheric modifications, Phys. Lett. A, 305, 264-274, 2002.

Hibberd, F. H., Nielsen, E., Stubbe, P., Kopka, H., and Rietveld, M. T.: Production of auroral zone $E$ region irregularities by powerful HF heating, J. Geophys. Res., 88, 6347-6351, 1983.

Hoeg, P.: Directional changes in the irregularity drift during artificial generation of striations, Physica Scripta, 33, 469-474, 1986.

Hysell, D. L.: $30 \mathrm{MHz}$ radar observations of artificial $\mathrm{E}$ region field-aligned plasma irregularities, Ann. Geophys., 26, 117-129, 2008, http://www.ann-geophys.net/26/117/2008/.

Hysell, D. L. and Chau, J. L.: Optimal aperture synthesis radar imaging, Radio Sci., 41, RS2003, doi:10.1029/2005RS003383, 2006.

Hysell, D. L. and Nossa, E.: Artificial E-region field-aligned plasma irregularities generated at pump frequencies near the second electron gyroharmonic, Ann. Geophys., 27, 2711-2720, 2009, http://www.ann-geophys.net/27/2711/2009/.

Isham, B., Rietveld, M., Hagfors, T., LaHoz, C., Mishin, E., Kofman, W., and van Eyken, T. L. A.: Aspect angle dependence of HF enhanced ionospheric backscatter, Adv. Space Res., 24(8), 1003-1006, 1999.

Jones, T. B., Robinson, T., Stubbe, P., and Kopka, H.: A hysteresis effect in the generation of field-aligned irregularities by a highpower radio wave, Radio Sci., 18, 835-839, 1983.

Kopka, H., Stubbe, P., Jones, T. B., and Robinson, T.: Nonlinear reflectivity of high-power radio waves in the ionosphere, Nature, 295, 680, doi:10.1038/295,680a0, 1982.

Kosch, M. J., Rietveld, M. T., Hagfors, T., and Leyser, T. B.: High-latitude HF-induced airglow displaced equatorwards of the pump beam, Geophys. Res. Lett., 27(17), 2817-2820, doi:10.1029/2000GL003754, 2000.

Mishin, E. V., Kosch, M. J., Pedersen, T. R., and Burke, W. J.: HFinduced airglow at magnetic zenith: Thermal and parametric instabilities near electron gyroharmonics, Geophys. Res. Lett., 32, L23106, doi:10.1029/2005GL023864, 2005.

Noble, S. T., Djuth, F. T., Jost, R. J., Gordon, W. E., Hedberg, A., Thide, B., Derblom, H., Bostrom, R., Nielsen, E., Stubbe, P., and Kopka, H.: Multiple-frequency radar observations of high- 
latitude $E$ region irregularities in the HF modified ionosphere, J. Geophys. Res., 92, 13613-13627, 1987.

Pedersen, T., McCarrick, M., Gerken, E., Selcher, C., Sentman, D., and Gurevich, H. C. A.: Magnetic zenith enhancement of $\mathrm{HF}$ radio-induced airglow production at HAARP, Geophys. Res. Lett., 1169, doi:10.1029/2002GL016096, 2003.

Pedersen, T., Esposito, R., Starks, M., and McCarrick, M.: Quantitative determination of HF radio-induced optical emission production efficiency at high latitudes, J. Geophys. Res., 113, A11316, doi:10.1029/2008JA013502, 2008.

Pedersen, T. R. and Carlson, H. C.: First observations of HF heaterproduced airglow at the High Frequency Active Auroral Research Program facility: Thermal excitation and spatial structuring, Radio Sci., 36(5), 1013-1026, doi:10.1029/2000RS002399, 2001.

Rietveld, M., Kosch, M., Blagoveshchenskaya, N., Kornienko, V., Leyser, T., and Yoeman, T.: Ionospheric electron heating, optical emissions and striations induced by powerful HF radio waves at high latitudes: Aspect angle dependence, J. Geophys. Res., 108, 1141, doi:10.1029/2002JA009543, 2003.
Stubbe, P., Kopka, H., Lauche, H., Rietveld, M. T., Brekke, A., Holt, O., Jones, T. B., Robinson, T., Hedberg, A., Thide, B., Crochet, M., and Lotz, H. J.: Ionospheric modification experiments in northern Scandanavia, J. Atmos. Terr. Phys., 44, 1025-1041, 1982.

Tereshchenko, E. D., Yurik, R. Yu., Khudukon, B. Z., Rietveld, M. T., Isham, B., Belyey, V., Brekke, A., Hagfors, T., and Grill, M.: Directional features of the downshifted peak observed in HFinduced stimulated electromagnetic emission spectra obtained using an interferometer, Ann. Geophys., 24, 1819-1827, 2006, http://www.ann-geophys.net/24/1819/2006/.

Thidé, B. and Lundborg, B.: Structure of HF pump in ionospheric modification experiments, Physica Scripta, 33, 475-479, 1986.

Wright, D. M., Davies, J. A., Yeoman, T. K., Robinson, T. R., and Shergill, H.: Saturation and hysteresis effects in ionospheric modification experiments observed by the CUTLASS and EISCAT radars, Ann. Geophys., 24, 543-553, 2006, http://www.ann-geophys.net/24/543/2006/. 